\title{
Scattered data interpolation by box splines
}

\author{
Zouwei Shen* and Shayne Waldron
}

\begin{abstract}
Department of Mathematics, National University of Singapore, Lower Kent Ridge Rd, Singapore 119260 email: matzuows@leonis.nus.edu.sg (http://www.math.nus.edu.sg/〜matzuows)
\end{abstract}

Department of Mathematics, University of Auckland, Private Bag 92019, Auckland, New Zealand e-mail: waldron@math.auckland.ac.nz (http://www.math.auckland.ac.nz/ ${ }^{\sim}$ waldron)

\begin{abstract}
Given scattered data in $\mathbb{R}^{s}$, interpolation from a dilated box spline space $S_{M}\left(2^{k} \cdot\right)$ is always possible for a fine enough scaling. For example, for the Lagrange function of a point $\theta$ one could take any shifted dilate $M\left(2^{k} \cdot-j\right)$ which is nonzero at $\theta$ and zero at the other interpolation points. However, the resulting interpolant, though smooth (and local), will consist of a set of "bumps", and so by any reasonable measure provides a poor representation of the shape of the underlying function. On the other hand, it is possible to choose a space of interpolants which contains some $M\left(2^{k} \cdot-j\right)$ of arbitrarily large support. But the resulting methods are increasingly less local, and in general still require some splines with a much higher level of dilation. Here we provide a multilevel method which constructs a space of interpolants by taking as many splines as possible from a given dilation level, then as many from the next (higher) dilation level, and so forth. The choice at each level is made using the suggestion of [W99], which is based on the Riesz representation theorem. This requires an inner product on the ground space $S_{M}$, and the higher levels $S_{M}\left(2^{k} \cdot\right) \ominus S_{M}\left(2^{k-1} \cdot\right), k=1,2, \ldots$ The inner products used here involve the box spline coefficients, and prewavelet coefficients of [RS92], respectively, and are norm equivalent to $\|\cdot\|_{L_{2}\left(\mathbb{R}^{s}\right)}$. These lead to a scheme which is easily implemented, and numerically stable. Previously, box spline interpolants have been considered only for data on a regular grid.
\end{abstract}

Key Words: box spline, multilevel approximation, adaptive methods AMS (MOS) Subject Classifications: primary 65D05, 65D07 secondary 42C40,

* This research is partially supported by the Academic Research Fund RP3981647, National University of Singapore. 


\section{Introduction}

This paper deals with the interpolation of scattered data using box splines. Previously, only cardinal box spline interpolation (data on a regular grid) has been considered (see [BHR93] and [RS95] for fundamental solutions which are of exponential decay and compact support, respectively). The multilevel scheme proposed here is based on a generalisation of the least solution of the polynomial interpolation problem of de Boor and Ron [BR92] to an arbitrary inner product space of interpolants by Waldron [W99], and uses the box spline wavelet decomposition of Riemenschneider and Shen [RS92].

Given linear functionals $\lambda_{1}, \ldots, \lambda_{n}$ on some space $X$, in our case function evaluation at scattered data points, we say interpolation from a subspace $V$ is correct (aka unisolvent, poised, well posed, etc) if for each $f \in X$, there is a unique $g \in V$ with

$$
\lambda_{i}(g)=\lambda_{i}(f), \quad 1 \leq i \leq n,
$$

and we denote by $\mathcal{L}: X \rightarrow V$ the interpolation operator $f \mapsto g$.

Suppose that $V$ is a correct space contained in $P$ a space of possible interpolants, in our case $\bigcup_{k \geq 0} S_{M}\left(2^{k} \cdot\right)$, which is graded, i.e.,

$$
P_{k}:=H_{0} \oplus H_{1} \oplus \cdots \oplus H_{k} \subset P, \quad P=\bigcup_{k \geq 0} P_{k}
$$

where functions in $H_{k}$ are thought of as being simpler (lower degree), or more appropriate for the interpolation, than those from $H_{k+1}$. In practice $H_{k}$ will be a space of low computational complexity for small $k$. Following the example where $P_{k}$ is the space of polynomials of degree $\leq k$, we say that $\mathcal{L}$ is degree reducing if

$$
\mathcal{L}\left(P_{k}\right) \subset P_{k}, \quad k=0,1, \ldots,
$$

or, equivalently, the correct space $V$ is of minimal degree, i.e.,

$$
\operatorname{dim}\left(V \cap P_{k}\right)=\operatorname{dim}\left(\operatorname{span}\left\{\left.\lambda_{i}\right|_{P_{k}}\right\}\right), \quad k=0,1, \ldots
$$

If interpolation from $P$ is possible, then minimal degree interpolation spaces $V$ (those as simple as possible) always exist (see [W99]).

It is instructive to think of a minimal degree $V$ as being built up by taking as many (linearly independent) functions from $H_{0}$ as possible, then as many from $H_{1}$ as possible, and so forth. Unless all the $H_{k}$ are one dimensional, there are many ways to do this. If each $H_{k}$ has an inner product structure, then [W99] suggests taking from $H_{k}$ the subspace

$$
R_{P_{k}}\left(\operatorname{span}\left\{\lambda_{1}, \ldots, \lambda_{n}\right\}\right) \cap H_{k}, \quad k=0,1, \ldots,
$$


where $R_{H}(\Lambda)$ denotes the Riesz representation of the linear functionals $\Lambda$ defined on some Hilbert space $H$. This paper investigates the choice of $P_{k}$ as the dilated box spline space

$$
P_{k}:=S_{M}^{k}:=S_{M}\left(2^{k} \cdot\right):=\left\{f\left(2^{k} \cdot\right): f \in S_{M}\right\}
$$

and $H_{k}$ a suitably chosen complement of $P_{k-1}$ in $P_{k}$, i.e.,

$$
H_{0}:=S_{M}, \quad H_{k}:=S_{M}^{k} \ominus S_{M}^{k-1}, \quad k=1,2, \ldots
$$

Here $S_{M}$ is the closed shift invariant subspace of $L_{2}\left(\mathbb{R}^{s}\right)$ generated by a box spline $M$.

To obtain an implementable interpolation method it remains only to endow each $H_{k}$ with an inner product which is norm equivalent to $\|\cdot\|_{L_{2}\left(\mathbb{R}^{s}\right)}$, and for which the Riesz representations of the interpolation conditions $\left\{\lambda_{i}\right\}$ are easily computed. Our choice is detailed in the next section. Briefly, it involves choosing a finite number of functions which generate the shift invariant spaces $H_{k}$ ( $M$ for $H_{0}$, and wavelets for $k>0$ ), and taking the inner product in which the shifts of these are orthogonal.

Throughout the paper, we illustrate our construction with the representative univariate and bivariate examples of a cubic B-spline $M$ and the box spline $M_{222}$, respectively.

\section{Box spline and prewavelet inner product spaces}

The development of box splines outlined below closely follows the monograph [BHR93], which can be consulted for more detail.

\subsection{Box splines defined}

Given an $s \times n$ matrix $\Xi$ of rank $s$ with integer entries, the box spline $M_{\Xi}$ on $\mathbb{R}^{s}$ is defined via its Fourier transform as

$$
\widehat{M}_{\Xi}(\omega):=\prod_{\xi \in \Xi} \frac{1-\exp (-i \xi \omega)}{i \xi \omega}
$$

where $\xi \in \Xi$ denotes the $n$ columns $\xi \in \mathbb{Z}^{s}$ of $\Xi$, and $\xi \omega$ is the dot product of $\xi$ and $\omega$. The box spline $M_{\Xi}$ is made up of polynomial pieces of degree $n-s$, and belongs to $C^{r(\Xi)-1}\left(\mathbb{R}^{s}\right)$, where $r(\Xi)+1$ is the minimum number of columns that can be discarded from $\Xi$ to obtain a matrix of rank less than $s$. It is compactly supported on the polyhedron

$$
\Xi(\square)=\left\{\sum_{\xi \in \Xi} t_{\xi} \xi: 0 \leq t_{\xi} \leq 1\right\}, \quad \square:=[0,1]^{n}
$$


and is symmetric about the point

$$
c_{\Xi}:=\sum_{\xi \in \Xi} \xi / 2
$$

The box spline $M_{\Xi}$ satisfies the refinement equation

$$
M_{\Xi}=2^{s} \sum_{j \in \mathbb{Z}^{s}} m_{\Xi}(j) M_{\Xi}(2 \cdot-j)
$$

where its refinement mask $m_{\Xi}: \mathbb{Z}^{s} \rightarrow \mathbb{R}$ is given in terms of the Fourier series

$$
\widehat{m}_{\Xi}(\omega):=\sum_{j \in \mathbb{Z}^{s}} m_{\Xi}(j) \exp (-i j \omega):=\prod_{\xi \in \Xi} \frac{1+\exp (-i \omega \xi)}{2} .
$$

The dependence on $\Xi$ will not be indicated when it is clear from the context. The univariate box splines with $\Xi=[1,1, \ldots, 1]$ are the familiar B-splines.

\subsection{Shift invariant spaces $S\left(\phi_{1}, \ldots, \phi_{r}\right)$}

Let $S\left(\phi_{1}, \ldots, \phi_{r}\right)$ be the closed shift invariant subspace of $L_{2}\left(\mathbb{R}^{s}\right)$ generated by a set of compactly supported functions $\phi_{1}, \ldots, \phi_{r} \in L_{2}\left(\mathbb{R}^{s}\right)$, and denote by $S_{M}:=S(M)$ the space generated by a single box spline $M=M_{\Xi}$. The $\phi_{i}$ are said to be stable if their shifts form a Riesz basis for $S\left(\phi_{1}, \ldots, \phi_{r}\right)$, i.e., there exists $C_{1}, C_{2}>0$ with

$$
C_{1} \sum_{i=1}^{r}\left\|a_{i}\right\|_{\ell_{2}\left(\mathbb{Z}^{s}\right)} \leq\left\|\sum_{i=1}^{r} \sum_{j \in \mathbb{Z}^{s}} \phi_{i}(\cdot-j) a_{i}(j)\right\|_{L_{2}\left(\mathbb{R}^{s}\right)} \leq C_{2} \sum_{i=1}^{r}\left\|a_{i}\right\|_{\ell_{2}\left(\mathbb{Z}^{s}\right)}, \quad \forall a_{i} \in \ell_{2}\left(\mathbb{Z}^{s}\right) .
$$

When $\phi_{1}, \ldots, \phi_{r}$ are stable, the only case we will consider,

$$
S\left(\phi_{1}, \ldots, \phi_{r}\right)=\left\{\sum_{i=1}^{r} \sum_{j \in \mathbb{Z}^{s}} \phi_{i}(\cdot-j) a_{i}(j): a_{i}(j) \in \mathbb{R}, \sum_{i, j}\left|a_{i}(j)\right|^{2}<\infty\right\} \subset L_{2}\left(\mathbb{R}^{s}\right)
$$

Sums such as those in (2.2), can be written as the convolution of a function with a sequence

$$
\phi * a:=\sum_{j \in \mathbb{Z}^{s}} \phi(\cdot-j) a(j), \quad \phi: \mathbb{R}^{s} \rightarrow \mathbb{R}(\text { compactly supported }), \quad a: \mathbb{Z}^{s} \rightarrow \mathbb{R},
$$

which has Fourier transform

$$
\widehat{\phi * a}=\widehat{\phi} \widehat{a}, \quad \widehat{a}(\omega):=\sum_{j \in \mathbb{Z}^{s}} a(j) \exp (-i j \omega) .
$$


We also use the semidiscrete convolution of a compactly supported $\phi$ with any $f: \mathbb{R}^{s} \rightarrow \mathbb{R}$

$$
\phi *^{\prime} f:=\phi *^{\prime}\left(\left.f\right|_{\mathbb{Z}^{s}}\right)=\sum_{j \in \mathbb{Z}^{s}} \phi(\cdot-j) f(j)
$$

which has Fourier transform

$$
\widehat{\phi *^{\prime}} f=\widehat{\phi} \tilde{f}, \quad \tilde{f}(\omega):=\sum_{j \in \mathbb{Z}^{s}} f(j) \exp (-i j \omega)
$$

When $\phi_{1}, \ldots, \phi_{r}$ are stable, we endow $S:=S\left(\phi_{1}, \ldots, \phi_{r}\right)$ with the inner product

$$
\langle f, g\rangle=\left\langle\sum_{i} \phi_{i} * a_{i}, \sum_{j} \phi_{j} * b_{j}\right\rangle:=\sum_{i}\left\langle a_{i}, b_{i}\right\rangle
$$

in which the shifts of $\phi_{1}, \ldots, \phi_{r}$ are orthogonal. This is norm equivalent to $\|\cdot\|_{L_{2}\left(\mathbb{R}^{s}\right)}$.

\subsection{The representation of linear functionals on $S\left(\phi_{1}, \ldots, \phi_{r}\right)$}

Our scheme is based on the Riesz representations of (compactly supported) linear functionals on $S:=S\left(\phi_{1}, \ldots, \phi_{r}\right)$, such as point evaluations, with respect to the inner product (2.3), for which the shifts of $\phi_{1}, \ldots, \phi_{r}$ are orthogonal.

We say that a linear functional $\lambda: S \mapsto \mathbb{R}$ is compactly supported if each of the sequences

$$
a_{i}: \mathbb{Z}^{s} \rightarrow \mathbb{R}: j \mapsto \lambda\left(\phi_{i}(\cdot-j)\right), \quad 1 \leq i \leq r
$$

has compact support. Clearly every compactly supported distribution has this property when restricted to $S$. Each compactly supported linear functional $\lambda: S \mapsto \mathbb{R}$ has a unique Riesz representation

$$
\lambda(f)=\langle f, g\rangle, \quad \forall f \in S,
$$

where $\langle\cdot, \cdot\rangle$ is the inner product $(2.3)$, and $g \in S$ is the compactly supported function defined by

$$
g=R_{S}(\lambda):=\sum_{i} \phi_{i} * a_{i}, \quad a_{i}(j):=\lambda\left(\phi_{i}(\cdot-j)\right)
$$

Example 1. For continuous $\phi_{i}$, point evaluation at any $\theta \in \mathbb{R}^{s}$, i.e., $\lambda=\delta_{\theta}: f \mapsto f(\theta)$, is a compactly supported linear functional on $S=S\left(\phi_{1}, \ldots, \phi_{r}\right)$, and so has a (compactly supported) representer $R_{S}\left(\delta_{\theta}\right) \in S$ given by (2.5). For example, when $S=S_{M}$, we compute

$$
g_{\theta}:=R_{S}\left(\delta_{\theta}\right):=M *^{\prime} M(\theta-\cdot)=\sum_{\substack{j \in \mathbb{Z}^{s} \\ M(\theta-j) \neq 0}} M(\cdot-j) M(\theta-j) .
$$

Notice that the spline $g_{\theta}$, a convex combination of shifts of $M$, is nonnegative, with compact support (which is easily calculated), satisfies $g_{\theta+j}=g_{\theta}(\cdot-j), j \in \mathbb{Z}^{s}$, and integrates to 1 . 
Moreover, $g_{\theta}$ is centred around $\theta$, and the map $\theta \mapsto g_{\theta}$ is continuous. For example, let $M_{j}$ be the cubic B-spline centred at $j$, then for $0 \leq \theta \leq 1$ we compute

$$
g_{\theta}=\frac{1}{6}(1-\theta)^{3} M_{-1}+\left(\frac{2}{3}-\frac{1}{2} \theta^{2}(2-\theta)\right) M_{0}+\left(\frac{2}{3}-\frac{1}{2}(1-\theta)^{2}(\theta+1)\right) M_{1}+\frac{1}{6} \theta^{3} M_{2} .
$$
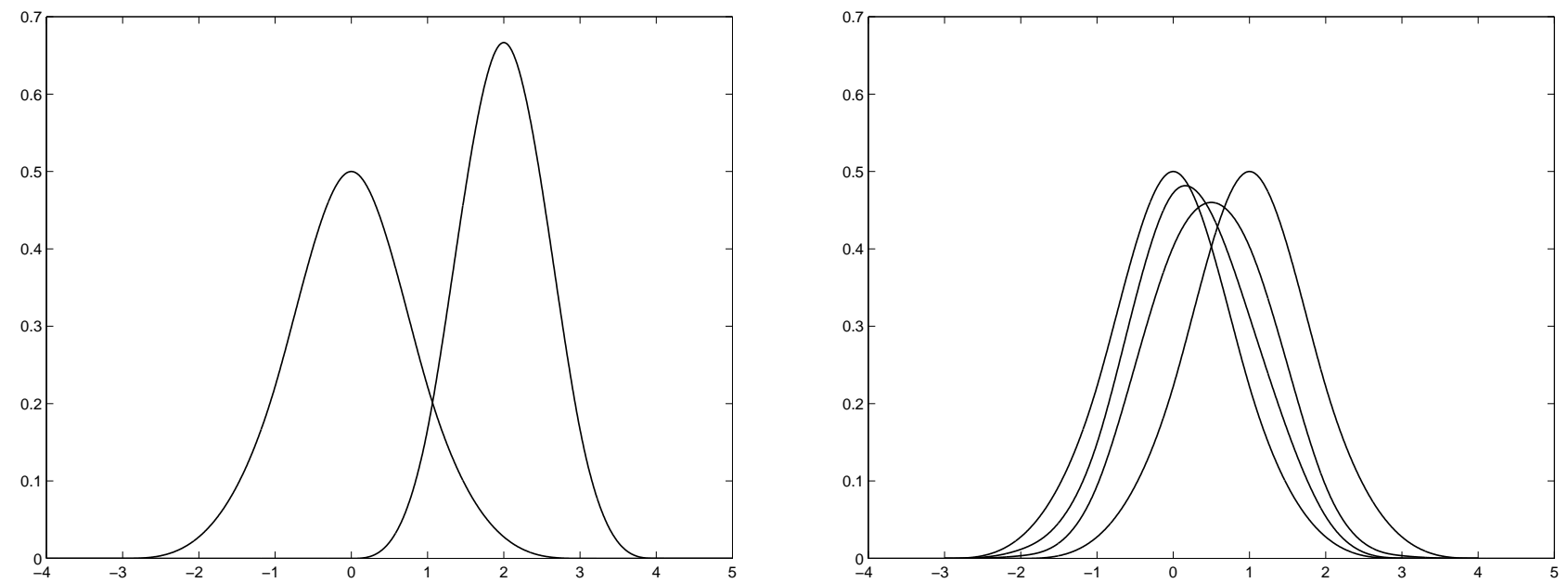

Fig. 2.1. The first figure shows the cubic B-spline $M$ with support [0,4], together with the corresponding Riesz representer $g_{0}$ of point evaluation at the origin, which has support $[-3,3]$. The second illustrates the continuity of the map $g \mapsto g_{\theta}$ by giving the Riesz representers $g_{0}, g_{0.25}, g_{0.5}, g_{1}$.
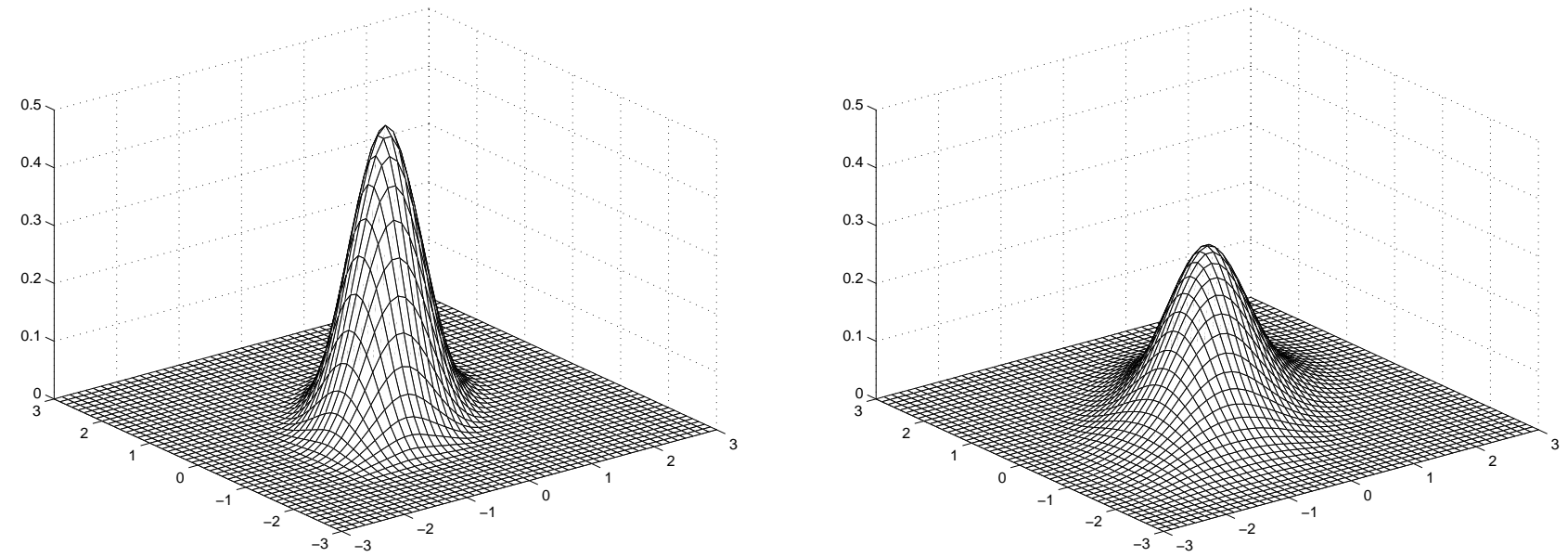

Fig. 2.2. The centered box spline $M_{222}^{c}$, and the corresponding Riesz representer of point evaluation at the origin.

Example 2. The value of the derivative at $\theta \in \mathbb{R}$, i.e., $\lambda=\delta_{\theta}(D): f \mapsto f^{\prime}(\theta)$ is a compactly supported linear functional on any B-spline space of degree $\geq 2$, in which case from (2.5) and (2.6) we have

$$
R_{S}\left(\delta_{\theta}(D)\right)=\frac{d}{d \theta} g_{\theta}
$$


In particular, for our cubic spline example

$$
R_{S}\left(\delta_{\theta}(D)\right)=-\frac{1}{2}(1-\theta)^{2} M_{-1}-\frac{1}{2} \theta(4-3 \theta) M_{0}+\frac{1}{2}(1-\theta)(3 \theta+1) M_{1}+\frac{1}{2} \theta^{2} M_{2} .
$$

Similarly, for the second derivative

$$
R_{S}\left(\delta_{\theta}\left(D^{2}\right)\right)=(1-\theta) M_{-1}+(3 \theta-2) M_{0}+(1-3 \theta) M_{1}+\theta M_{2},
$$

and for the third derivative, which is not defined at 0 and 1 , we have

$$
R_{S}\left(\delta_{\theta}\left(D^{3}\right)\right)=-M_{-1}+3 M_{0}-3 M_{1}+M_{2}, \quad 0<\theta<1 .
$$

The property (2.7) extends, in the obvious fashion, to $\lambda$ any derivative that is defined on the given box spline space $S_{M}$.
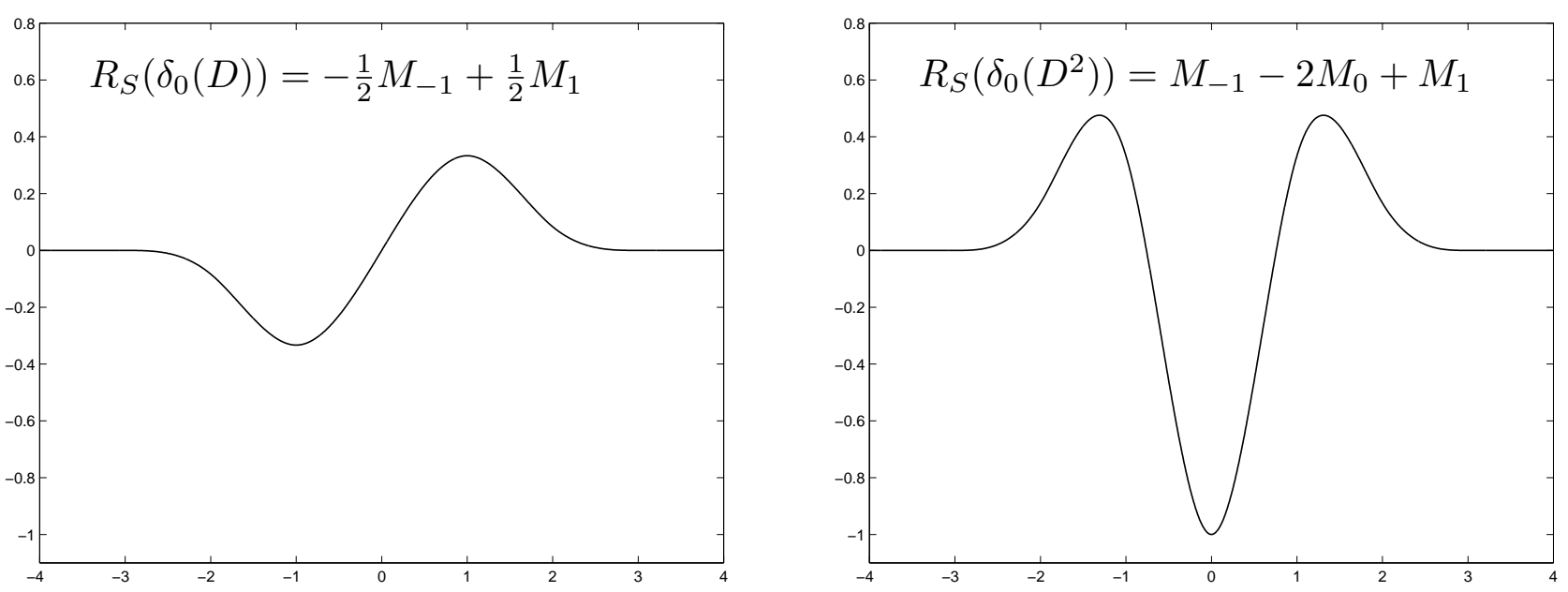

Fig. 2.3. The cubic B-spline representers of the first and second derivatives at 0 .

\subsection{Box spline prewavelets and semiwavelets}

The refinability of (2.1) implies $S_{M}^{k} \subset S_{M}^{k+1}$, and so the grading (1.1) is well defined. Each $H_{k}:=S_{M}^{k} \ominus S_{M}^{k-1}$ is a dilate of the finitely generated shift invariant space $H_{1}(\cdot / 2)$.

We endow $H_{0}=S(M)$ with the inner product (2.3), and restrict ourselves to the case when this is norm equivalent to $\|\cdot\|_{L_{2}\left(\mathbb{R}^{s}\right)}$, i.e., $M$ is stable. This occurs when $\Xi$ is a unimodular matrix, i.e., all bases of columns from $\Xi$ have determinant \pm 1 , which is equivalent to there being no $\omega \in \mathbb{R}^{s}$ at which $\widehat{M}(\omega+2 \pi j)=0, \forall j \in \mathbb{Z}^{s}$. Thus, the shifts of the box spline $M_{\Xi}$ form a Riesz basis if and only if the autocorrelation function

$$
P(\omega)=P_{\Xi}(\omega):=\sum_{j \in \mathbb{Z}^{s}} M_{\Xi \cup-\Xi}(j) \exp (-i j \omega)=\sum_{j \in \mathbb{Z}^{s}}\left|\widehat{M}_{\Xi}(\omega+j)\right|^{2}
$$

is positive for all $\omega \in \mathbb{R}^{s}$. 
For $H_{k+1}=H_{1}\left(2^{k} \cdot\right)$, we also take the inner product $(2.3)$, via

$$
H_{1}=S\left(\psi_{1}, \ldots, \psi_{2^{s}-1}\right)
$$

where the prewavelets $\psi_{1}, \ldots, \psi_{2^{s}-1}$ (whose shifts form a Riesz basis) are now described.

Here we follow the development of [RS92]. Let $\mathbb{Z}_{2}^{s}$ be the quotient group $\mathbb{Z}^{s} / 2 \mathbb{Z}^{s}$. Given a refinement mask $m=m_{\Xi}$, for each $\mu \in \mathbb{Z}_{2}^{s} \backslash\{0\}$, define

$$
\widehat{b}_{\mu}(\omega):=\exp (i \eta(\mu) \omega) \begin{cases}P(\omega+\pi \mu) \widehat{m}(\omega+\pi \mu), & 2 c_{\Xi} \mu \text { is odd } \\ P(\omega+\pi \mu) \widehat{m}(\omega+\pi \mu), & 2 c_{\Xi} \mu \text { is even }\end{cases}
$$

where the map $\eta: \mathbb{Z}_{2}^{s} \rightarrow \mathbb{Z}_{2}^{s}$ is defined by $\eta(0)=0$ and $\eta(1)=1$ when $s=1$, and for $s=2$ as follows

$$
\eta:(0,0) \mapsto(0,0),(0,1) \mapsto(0,1),(1,0) \mapsto(1,1),(1,1) \mapsto(1,0)
$$

In [RS92] it was shown that the $2^{s}-1$ prewavelets $\psi_{\mu}, \mu \in \mathbb{Z}_{2}^{s} \backslash\{0\}$, defined by

$$
\widehat{\psi}_{\mu}(2 \omega):=\widehat{b}_{\mu}(\omega) \widehat{M}(\omega) \quad \Longleftrightarrow \quad \psi_{\mu}=M * b_{\mu}
$$

and their shifts form a Riesz basis for $H_{1}$. Furthermore, the system

$$
\left\{2^{j / 2} \psi_{\mu}\left(2^{j} \cdot-\alpha\right): j \in \mathbb{Z}, \mu \in \mathbb{Z}_{2}^{s} \backslash\{0\}, \alpha \in \mathbb{Z}^{s}\right\}
$$

is a Riesz basis of $L_{2}\left(\mathbb{R}^{s}\right)$.

Also available are the semiwavelets $\Psi_{\mu}, \mu \in \mathbb{Z}_{2}^{s} \backslash\{0\}$, defined by

$$
\widehat{\Psi}_{\mu}(2 \omega):=\widehat{m}_{\mu}(\omega) \widehat{M}(\omega) \quad \Longleftrightarrow \quad \Psi_{\mu}=M * m_{\mu}
$$

where

$$
\widehat{m}_{\mu}(\omega):=\exp (i \eta(\mu) \omega) \begin{cases}\overline{\widehat{m}(\omega+\pi \mu)}, & 2 c_{\Xi} \mu \text { is odd; } \\ \widehat{m}(\omega+\pi \mu), & 2 c_{\Xi} \mu \text { is even. }\end{cases}
$$

These semiwavelets have smaller support than the prewavelets. The space $S\left(\left\{\Psi_{\mu}\right\}\right)$ is an algebraic (but not orthogonal) complement of $S_{M}$ in $S_{M}(2 \cdot)$, and the functions

$$
\left\{2^{j / 2} \Psi_{\mu}\left(2^{j} \cdot-\alpha\right): 0 \leq j \leq k, \mu \in \mathbb{Z}_{2}^{s} \backslash\{0\}, \alpha \in \mathbb{Z}^{s}\right\}
$$

form a Riesz system. It is as yet unknown whether with $j$ unrestricted they provide a Riesz basis for $L_{2}\left(\mathbb{R}^{s}\right)$. Hence, the semiwavelet system might yield a less stable algorithm when a large number of levels have to be used to obtain the interpolant. 


\subsection{Examples}

We will represent finitely supported maps $\mathbb{Z} \rightarrow \mathbb{R}, \mathbb{Z}^{2} \rightarrow \mathbb{R}$ as vectors and matrices of their nonzero values, with the value at 0 in bold and the usual indexing.

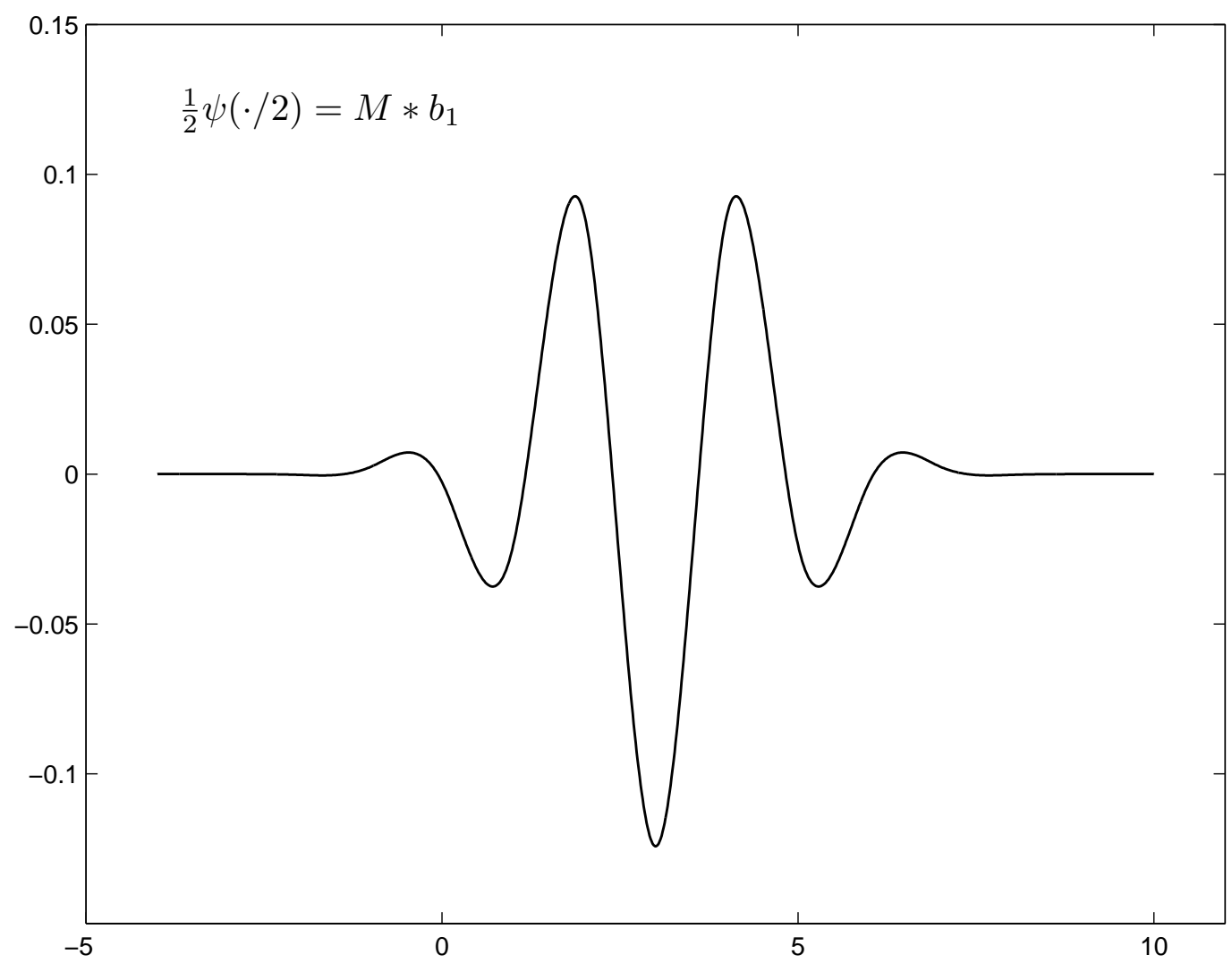

Fig. 2.4. The cubic prewavelet $\psi(\cdot / 2)$. This has support $[-4,10]$, and is symmetric about 3 .

Example (Cubic spline wavelets). The choice $\Xi=[1,1,1,1]$ gives the cubic B-spline

$$
\begin{gathered}
\widehat{M}(\omega)=\left(\frac{1-e^{-i \omega}}{\omega}\right)^{4}, \quad \operatorname{supp}(M)=\Xi(\square)=[0,4], \quad c_{\Xi}=\frac{1}{2}(1+1+1+1)=2, \\
\widehat{m}(\omega)=\left(\frac{1+e^{-i \omega}}{2}\right)^{4}=\frac{1}{16} e^{-4 i \omega}+\frac{1}{4} e^{-3 i \omega}+\frac{3}{8} e^{-2 i \omega}+\frac{1}{4} e^{-i \omega}+\frac{1}{16},
\end{gathered}
$$

which gives the refinement mask

$$
m=\frac{1}{16}[\mathbf{1}, 4,6,4,1]
$$

Evaluating the centred B-spline of order 8 at the integers gives

$$
\left.M_{\Xi \cup-\Xi}\right|_{\mathbb{Z}}=\frac{1}{5040}[1,120,1191, \mathbf{2 4 1 6}, 1191,120,1]
$$


and so the autocorrelation function is

$$
P(\omega)=\frac{1}{5040}\left(e^{-3 i \omega}+120 e^{-2 i \omega}+1191 e^{-i \omega}+2416+1191 e^{i \omega}+120 e^{2 i \omega}+e^{3 i \omega}\right) .
$$

Since $\mathbb{Z}_{2} \backslash\{0\}$ has one element $\mu=1$, and $2 c_{\Xi} \mu=4$ is even, we have

$$
\widehat{b}_{1}(\omega)=e^{i \omega} P(\omega+\pi) \widehat{m}(\omega+\pi),
$$

which gives

$$
b_{1}=\frac{1}{80640}[-1,124,-1677,7904,-\mathbf{1 8 4 8 2}, 24264,-18482,7904,-1677,124,-1] \text {. }
$$

Hence the prewavelet is

$$
\frac{1}{2} \psi(\cdot / 2)=\frac{1}{-} \psi_{1}(\cdot / 2)=M *^{\prime} b_{1} .
$$

As predicted by [RS92], this function is symmetric about

$$
c_{\mu}:=c_{\Xi}\left(1+(-1)^{2 c_{\Xi} \mu}\right)-\eta(\mu)=2\left(1+(-1)^{4}\right)-1=3 .
$$

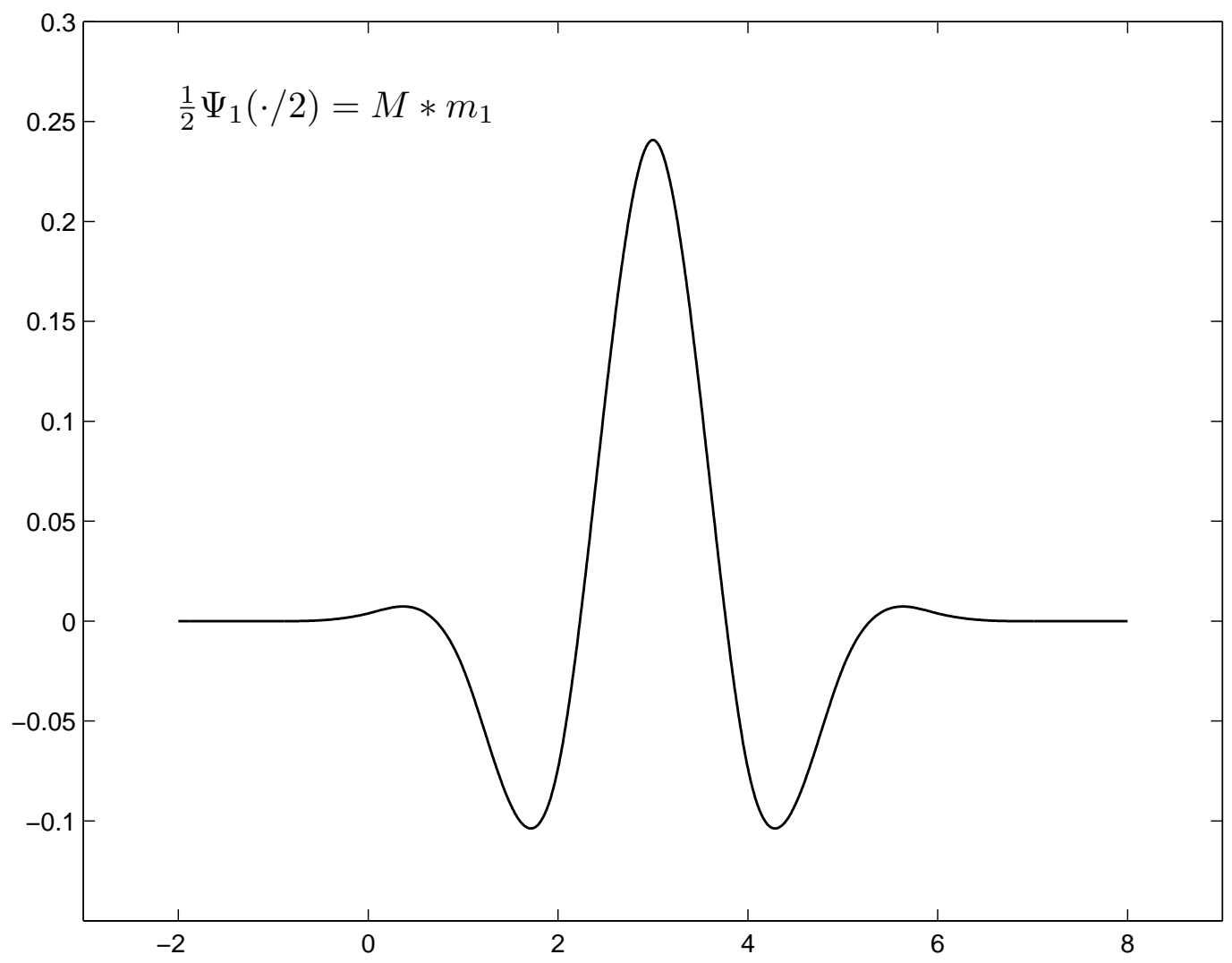

Fig. 2.5. The cubic semiwavelet $\Psi_{1}(\cdot / 2)$ (with smaller support than the prewavelet). 
The corresponding semiwavelet $\Psi_{1}$ of $(2.9)$ is given by the mask

$$
m_{1}=\frac{1}{5040}[-1,120,-\mathbf{1 1 9 1}, 2416,-1191,120,-1]
$$

Example $\left(M_{222}\right.$ box spline). Let $M_{222}$ be the box spline corresponding to the matrix

$$
\Xi:=\left(\begin{array}{llllll}
1 & 1 & 0 & 0 & 1 & 1 \\
0 & 0 & 1 & 1 & 1 & 1
\end{array}\right)
$$

This function is $C^{2}$, stable, and has centre $(2,2)$. Let $\phi:=M_{222}(\cdot-(2,2))$, which has the refinement mask

$$
m:=\frac{1}{64}\left(\begin{array}{ccccc}
0 & 0 & 1 & 2 & 1 \\
0 & 2 & 6 & 6 & 2 \\
1 & 6 & \mathbf{1 0} & 6 & 1 \\
2 & 6 & 6 & 2 & 0 \\
1 & 2 & 1 & 0 & 0
\end{array}\right)
$$

The sequence $a:=\left.(\phi * \phi(-\cdot))\right|_{\mathbb{Z}_{2}^{s}}$ is

$$
a=\frac{1}{362880}\left(\begin{array}{ccccccc}
0 & 0 & 0 & 2 & 34 & 34 & 2 \\
0 & 0 & 34 & 1736 & 5100 & 1736 & 34 \\
0 & 34 & 5100 & 37742 & 37742 & 5100 & 34 \\
2 & 1736 & 37742 & \mathbf{9 4 9 9 2} & 37742 & 1736 & 2 \\
34 & 5100 & 37742 & 37742 & 5100 & 34 & 0 \\
34 & 1736 & 5100 & 1736 & 34 & 0 & 0 \\
2 & 34 & 34 & 2 & 0 & 0 & 0
\end{array}\right) .
$$

Hence the prewavelet masks are

$$
b_{\mu}(\alpha):=(-1)^{\alpha \cdot \mu+1} b(\alpha+\eta(\mu)), \quad \mu \in \mathbb{Z}_{2}^{2},
$$

where $b=a * m$ is the convolution $b(i):=\sum_{j} a(i-j) m(j)$.

The corresponding semiwavelets are given by

$$
\Psi_{\mu}=2^{s} \sum_{\alpha \in \mathbb{Z}^{s}} m_{\mu}(\alpha) M_{222}(2 \cdot-\alpha), \quad \mu \in \mathbb{Z}_{2}^{2} \backslash\{0\},
$$

where

$$
m_{\mu}(\alpha):=(-1)^{\alpha \cdot \mu+1} m(\alpha+\eta(\mu)) .
$$




\subsection{Evaluation of box splines and wavelets}

To implement of our method we require the values of box splines and their corresponding prewavelets and semiwavelets, which can be computed as follows.

Let $m$ be the refinement mask of the box spline $M$, i.e.,

$$
M=\sum_{\alpha \in \mathbb{Z}} m(\alpha) M(2 \cdot-\alpha)
$$

and $m_{\mu}$ be the mask of the prewavelet (or semiwavelet) $\psi_{\mu}, \mu \in \mathbb{Z}_{2}^{s} \backslash\{0\}$, i.e.,

$$
\psi_{\mu}=\sum_{\alpha \in \mathbb{Z}} m_{\mu}(\alpha) M(2 \cdot-\alpha)
$$

Using (2.11) and (2.12) the values of $M$ and $\psi_{\mu}$ at dyadic points $\cup_{j \geq 0} 2^{-j} \mathbb{Z}^{s}$ can be calculated from the value of $M$ at the integers $\mathbb{Z}^{s}$. This can be done as follows.

Suppose the support of $M$ is contained in $[0, N]^{s}$, and define the matrix

$$
\mathbb{M}:=\left[2^{s} m(2 j-k)\right]_{j \in I, k \in I}, \quad I:=[0, N]^{s} \cap \mathbb{Z}^{s} .
$$

For the $M$ considered here, the matrix $\mathbb{M}$ has a unique dominant eigenvalue of 1 , which is simple, and the corresponding eigenvector is $\left.M\right|_{I \times I}$ (see e.g., [LLS97]). The entries of $\left.M\right|_{I \times I}$ sum to one. Once the values of $M$ are known at the integers, the values of $M$ and $\psi_{\mu}$ at dyadic points can be calculated exactly using (2.11) and (2.12), and hence as closely as desired at any point. For the $M_{222}$ box spline its values at the integers are given by

$$
M_{222}(j)= \begin{cases}1 / 2, & j=(2,2) ; \\ 1 / 12, & j \in\{(1,1),(2,1),(1,2),(2,3),(3,2),(3,3)\} \\ 0, & \text { otherwise. }\end{cases}
$$

Similarly, by taking the derivative of (2.11) and (2.12), it is easily seen that the values of a derivative of $M$ and $\psi_{\mu}$ can be reduced to the calculation of the integer values of the derivative of $M$. Since

$$
D_{\xi} M_{\Xi}=M_{\Xi \backslash \xi}-M_{\Xi \backslash \xi}(\cdot-\xi), \quad \xi \in \Xi,
$$

where $D_{\xi}$ is the derivative in the direction $\xi$, the integer values of $D_{\xi} M_{\Xi}$ can be obtained from those of $M_{\Xi \backslash \xi}$. Since the columns of $\Xi$ contain a basis of $\mathbb{R}^{s}$, it is possible to compute derivatives in any direction, and higher order derivatives can also be calculated. Finally, since all the other basis functions used in the calculation are linear combinations of box spline and its shifts, their valuse can be computed from those of box splines. 


\section{The least level method}

We now give details of the implementation of our "least level method" for interpolation.

\subsection{Overview}

Our objective is to interpolate function values (and possibly derivatives) at scattered points $\Theta \subset \mathbb{R}^{s}$ using splines from an appropriately chosen subspace of $S_{M}\left(2^{k} \cdot\right)$. This can always be done in a naive way by choosing a large $k$, then taking as the interpolant to $f$

$$
g:=\sum_{\theta \in \Theta} \frac{f(\theta)}{M\left(2^{k} \theta-j_{\theta}\right)} M\left(2^{k} \cdot-j_{\theta}\right),
$$

where the shifts $j_{\theta} \in \mathbb{Z}^{s}$ are such that $M\left(2^{k} \cdot-j_{\theta}\right)$ is zero at all points of $\Theta$ except $\theta$. For large $k$ the resulting interpolants though smooth (and local) consist of "bumps" near each of the interpolation points, and so provide a poor representation of the underlying function. On the other hand, it is possible to choose a space of interpolants which contains some $M\left(2^{k} \cdot-j\right)$ of arbitrarily large support, but such interpolants are increasingly less local, and still require some splines with a much higher level of dilation.

The least level method balances these factors by taking as many functions as possible from a low dilation level, then the remainder from increasingly higher levels. The starting level must be chosen in an appropriate way. The philosophy behind such a choice is the following. If a space of interpolants can be taken from just the starting level, then this level is highly dilated and the interpolants will be too localised (the situation of "bumps"), and one should start with a less dilated space. If functions with a much higher level of dilation than those in the starting level are required, then the starting level is not sufficiently dilated. In practice, we will start with a dilation level which would be appropriate for cardinal interpolation if the points were regularly spaced, and most of the interpolating space will come from this level. There will be a small contribution from higher levels which can be thought of as a correction which takes account of the scattering.

The nature of the data also influences the quality of the approximation, as illustrated in Section 4. For rapidly varying data a starting level with a higher dilation gives a better fit, while for smoother data a less dilated starting level gives better results.

A recent paper of Johnson [J99] considers the same problem of selecting a good interpolant to scattered data from a suitably dilated space $S(\phi)$, with $\phi$ a compactly supported function satisfying the Strang-Fix conditions. He shows that interpolants which "nearly minimise' a cost functional provide good approximation. This cost functional becomes large as a function is dilated, and so this selection method is similar in spirit to our own, where most of the interpolant is taken from a low dilation level. 


\subsection{Construction of the multilevel spline space}

To simplify the exposition we suppose the starting level is $H_{0}=S_{M}$, and that the points are chosen from some region $\Omega \subset \mathbb{R}^{s}$. Changing the starting level is equivalent to scaling $\Omega$, which we will assume has already been done appropriately.

The inner product on $H_{0}$ is such that $\left\{M(\cdot-j): j \in \mathbb{Z}^{s}\right\}$ is an orthonormal basis, and this choice is a Riesz basis for $L_{2}\left(\mathbb{R}^{s}\right)$. The higher levels $H_{i}=S_{M}^{i} \ominus S_{M}^{i-1}, i=1,2, \ldots$, are dilates of $S\left(\psi_{\mu}: \mu \in \mathbb{Z}_{2}^{s} \backslash\{0\}\right)$, namely

$$
H_{i}=\left\{\sum_{\mu \in \mathbb{Z}_{2}^{s} \backslash\{0\}} \sum_{j \in \mathbb{Z}^{s}} \psi_{\mu}\left(2^{i} \cdot-j\right) a_{\mu}(j): \sum_{\mu, j}\left|a_{\mu}(j)\right|^{2}<\infty\right\},
$$

where $\left\{\psi_{\mu}\right\}$ are either the prewavelets or semiwavelets of Section 2. An orthonormal basis

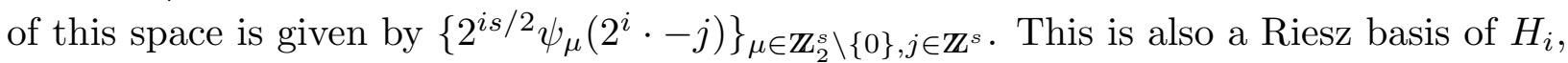
and the constants in the norm equivalence can be chosen independently of $i$ when the prewavelets are used. The Riesz basis property ensures the stability of our calculations which are done using the aforementioned bases, and only involve finite sequences of coefficients.

Using these orthogonal bases the Riesz representation of a (compactly supported) linear functional $\lambda$ defined on the orthogonal direct sum $P_{k}=S_{M}\left(2^{k} \cdot\right)=H_{0} \oplus \cdots \oplus H_{k}$ can be written as

$$
R_{P_{k}}(\lambda)=R_{H_{0}}(\lambda)+\cdots+R_{H_{k}}(\lambda)
$$

where, by (2.5),

$$
\begin{aligned}
R_{H_{0}}(\lambda) & =\sum_{j \in \mathbb{Z}^{s}} \lambda(M(\cdot-j)) M(\cdot-j), \\
R_{H_{i}}(\lambda) & =\sum_{\mu \in \mathbb{Z}_{2}^{s} \backslash\{0\}} \sum_{j \in \mathbb{Z}^{s}} \lambda\left(2^{i s / 2} \psi_{\mu}\left(2^{i} \cdot-j\right)\right) 2^{i s / 2} \psi_{\mu}\left(2^{i} \cdot-j\right), \quad i=1, \ldots, k .
\end{aligned}
$$

Our construction uses the functions given by (3.2), where the $\lambda$ are the functionals being matched. Since these functionals have support contained in $\Omega$, all but a finite number of the coefficients in (3.2) are zero, the relevant ones being those where

$$
\operatorname{supp}(M(\cdot-j)) \cap \Omega \neq\{\}, \quad \operatorname{supp}\left(\psi_{\mu}\left(2^{i} \cdot-j\right)\right) \cap \Omega \neq\{\}
$$

respectively. The only calculations used are vector space operations on the $R_{H_{i}}(\lambda)$, which will be performed using the vectors of relevant coefficients from (3.2). As already discussed, these calculations are stable.

Let $\lambda_{1}, \ldots, \lambda_{n}$ be the $n$ linear functionals that are to be matched. For us these are the point evaluations $f \mapsto(\theta), \theta \in \Theta$, though the method is quite general, and could be applied to any $\lambda_{i}$ with compact support such as derivative values (Hermite interpolation). 
We now construct the minimal degree interpolation space $V$ (multilevel spline space) using the method of [W99], which is effectively Gauss elimination applied to a matrix with entries from a vector space. For each $\lambda_{i}$, let

$$
h_{i k}:=R_{H_{k}}\left(\left.\lambda_{i}\right|_{H_{k}}\right) \in H_{k}, \quad k=0,1, \ldots,
$$

so that (3.1) becomes

$$
R_{P_{k}}\left(\left.\lambda_{i}\right|_{P_{k}}\right)=h_{i 0}+h_{i 1}+h_{i 2}+\cdots+h_{i k}
$$

and the i-th row of $A:=\left[h_{i j}\right]$ represents $\lambda_{i}$

$$
A=\left(\begin{array}{cccc}
h_{10} & h_{11} & h_{12} & \cdots \\
h_{20} & h_{21} & h_{22} & \cdots \\
\vdots & \vdots & \vdots & \\
h_{n 0} & h_{n 1} & h_{n 2} & \cdots
\end{array}\right) \longleftrightarrow\left(\begin{array}{c}
\lambda_{1} \\
\lambda_{2} \\
\vdots \\
\lambda_{n}
\end{array}\right)=\Lambda
$$

To the matrix $A$ (and the vector $\Lambda$ ) apply Gauss elimination (by segments) to obtain a row echelon form (see [B94] and [W99]). The difference between this and the classical method is that since entries of a given column now come from a vector space (as opposed to the one dimensional space $\mathbb{R}$ ) some may be linearly independent, and so elementary row operations can not make all but one of them zero. In this case we allow the possibility of more than one pivot in a given column (all of which are linearly independent).

The row echelon form gives a new set of functionals with the same span as $\lambda_{1}, \ldots, \lambda_{n}$, and their Riesz representations. By the construction, the pivots are linearly independent. Those in the $k$-th column are a basis for the space

$$
V_{k}:=R_{P_{k}}\left(\operatorname{span}\left\{\lambda_{1}, \ldots, \lambda_{n}\right\}\right) \cap H_{k}
$$

and the $n$-dimensional space $V:=V_{0} \oplus V_{1} \oplus \cdots$ is a minimal degree interpolation space. This is easily seen by observing that the value of a new linear functional applied to a function from a column up to that in which its leading term occurs is given by the inner product of the function and this leading term. Pivoting strategies to minimise the errors in this calculation are suggested in [W99].

Now that the interpolation space has been identified the interpolant can be constructed in the usual way by solving a (Vandermonde) matrix system. We suggest that this is done by performing an elimination (back substitution) to an orthogonal reduced row echelon form of $A$ as discussed in [W99]. First apply elementary row operations to make the pivots $p_{1}, \ldots, p_{n}$ orthonormal (Gram-Schmidt within each grade). Then introduce zeros in all 
entries above the pivots in a given row to obtain linear functionals $\Lambda^{\perp}=\left[\lambda_{i}^{\perp}\right]=M \Lambda$ with $\lambda_{i}^{\perp}\left(p_{j}\right)=\delta_{i j}$. Thus, the interpolant to $f$ is given by

$$
\mathcal{L}_{\Lambda} f=\sum_{i=1}^{n} p_{i} \lambda_{i}^{\perp}(f) .
$$

In the special case where $H_{0}=S_{M}$, the Riesz representors of evaluation at grid points are shifts of each other

$$
R_{H_{0}}\left(\delta_{j}\right)=g_{j}=\phi(\cdot-j), \quad j \in \mathbb{Z}^{s}, \quad \phi:=M *^{\prime} M(-\cdot) .
$$

These shifts form a Riesz basis when the symbol

$$
\widetilde{M}(\omega):=\sum_{j \in \mathbb{Z}^{s}} M(j) e^{-i j \omega}
$$

does not vanish, and so $\widehat{\phi}=\widetilde{M}(-\cdot) \widehat{M}$ has no $2 \pi$-periodic zeros. Since any finite set of these shifts is linearly independent, a dimension count shows that the least level interpolation space to $\Theta \subset \mathbb{Z}^{s}$ only uses functions from the ground level, i.e., is given by

$$
V_{\Theta}:=\operatorname{span}\{\phi(\cdot-j): j \in \Theta\}
$$

Moreover, the Gramian matrix for this interpolation is sparse since $\phi$ has compact support.

\section{Examples}

Here we give some univariate and bivariate examples of our scheme that illustrate the effect of scaling the ground space (the "bump" phenomenon), and the behaviour of the interpolant at the end points. We also see that the nature of the data itself plays a role.

Interpolation from $S_{M}(\cdot / h)$ to data on the integer mesh $h Z^{s}$ is uniquely possible when $\widetilde{M}$ does not vanish, and has good approximation properties as $h \rightarrow 0$. This suggests the rule of thumb that when the interpolation points have a 'density' like that of $h \mathbb{Z}^{s}$, i.e.,

$$
\sup _{x \in \Omega} \inf _{\theta \in \Theta}\|x-\theta\| \approx h
$$

then the ground space should be $S_{M}(\cdot / h)$. A higher or lower starting level of dilation may be desirable for data which is varying rapidly and slowly, respectively. 


\subsection{Cubic spline example}

Here we illustrate our method using the cubic spline prewavelet. Consider interpolation at the 10 scattered points

$$
\Theta=\{0.1755,0.6241,1.5088,3.1686,4.3299,6.2966,7.5399,8.4326,8.6842,9.6802\}
$$

in the interval $\Omega=[0,10]$, to data which is varying rapidly, and varying slowly.
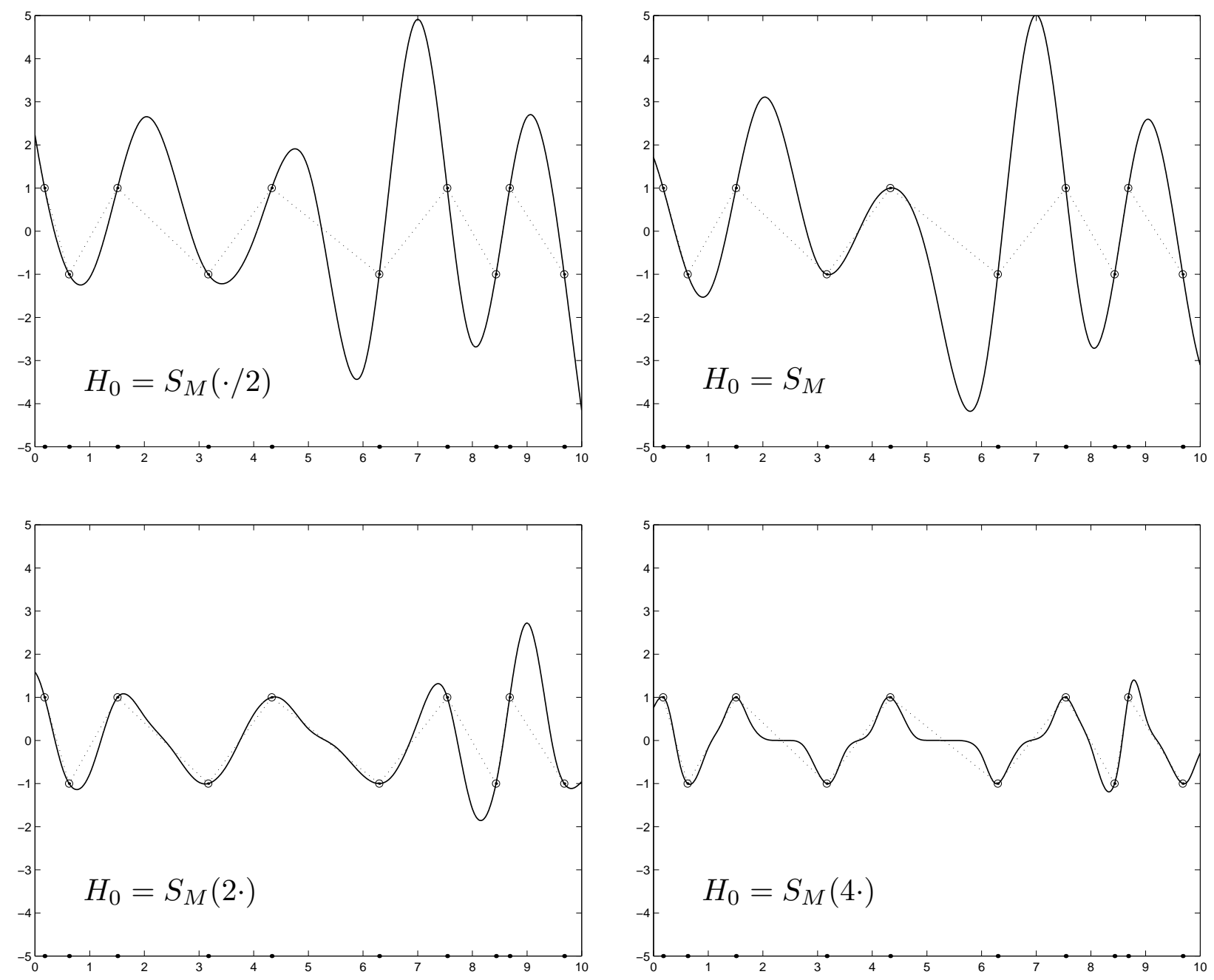

Fig. 4.1. The multilevel cubic spline interpolants to rapidly varying data \pm 1 at the 10 scattered points $\Theta$ in the interval $\Omega=[0,10]$. Notice the beginning of the "bump" effect for interpolation points with larger separations.

First we take as the ground space the cubic splines with breakpoints at the even integers, that is $H_{0}=S_{M}(\cdot / 2)$. The number of B-splines in this space with support intersecting $\Omega$ (and hence available for the interpolation space) is 8 , and all of this 8 -dimensional space is used. Since our prewavelet has support $[-4,10]$, there is a 23 -dimensional space available at the next level, and the remaining 2 dimensions are taken from it. 
Next we take the ground space with breakpoints at the integers, i.e., $H_{0}=S_{M}$. Here all of the interpolation space is taken from the ground level, where a 13-dimensional space is available. This is the suggested scaling.

In the third and fourth examples we take the ground space with breakpoints at the half and quarter integers, i.e., $S_{M}(2 \cdot)$ and $S_{M}(4 \cdot)$. Here the interpolation space is taken entirely from the ground level.
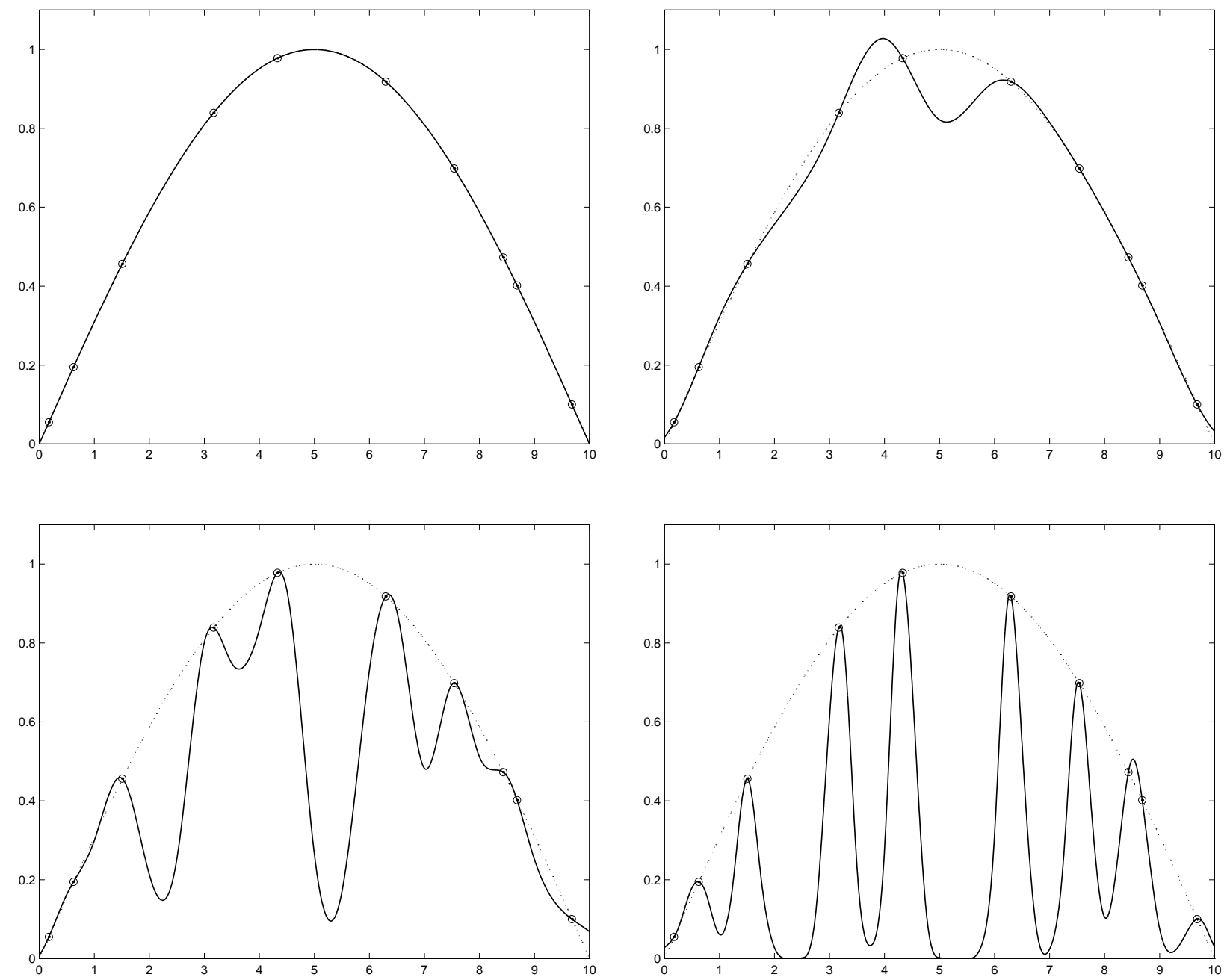

Fig. 4.2. The multilevel cubic spline interpolants to slowly varying data from one arc of $x \mapsto \sin (\pi x / 10)$ at the same 10 scattered points used in Fig. 4.1., with the same breakpoints. Notice the "bump" effect as the spline mesh becomes more dilated.

In Fig. 4.1., the third example provides perhaps the best fit, which shows that for rapidly varying data some over dilation of the ground space may be appropriate. On the other hand, the first example of Fig. 4.2. clearly gives the best fit, which indicates that for smoother data some under dilation of the ground space is desirable.

In all the calculations we performed the majority of the interpolation space came from 
the ground space when the scaling rule of thumb was adopted. Indeed, to find a set up where more than two levels was used it was necessary to "clump" points together to force the introduction of higher dilation wavelets to resolve them.

\subsection{Comparison with the classical cubic spline interpolants}

We compared our interpolant with the classical cubic spline interpolants in a variety of situations. The differences we observed relate to the behaviour at the boundary, and the "energy minimisation" properties of the cubic spline interpolant. The classical cubic spline interpolants give "minimal energy interpolants" to given data (see [B78:Ch.V]), whilst ours give interpolants with the simplest structure (as described in the introduction). Thus for problems where the interpolant should have minimal energy (the bending of plate for a car body) one should use the classical cubic splines, but where the data comes from an unknown function which does not have "low energy", we suggest, with appropriate scaling, that our interpolant might be more desirable. When data was given at the end points of $\Omega$, or the derivative is known at the first and last interpolation point so that the complete cubic spline interpolant can be used, then the classical methods invariably performed better.

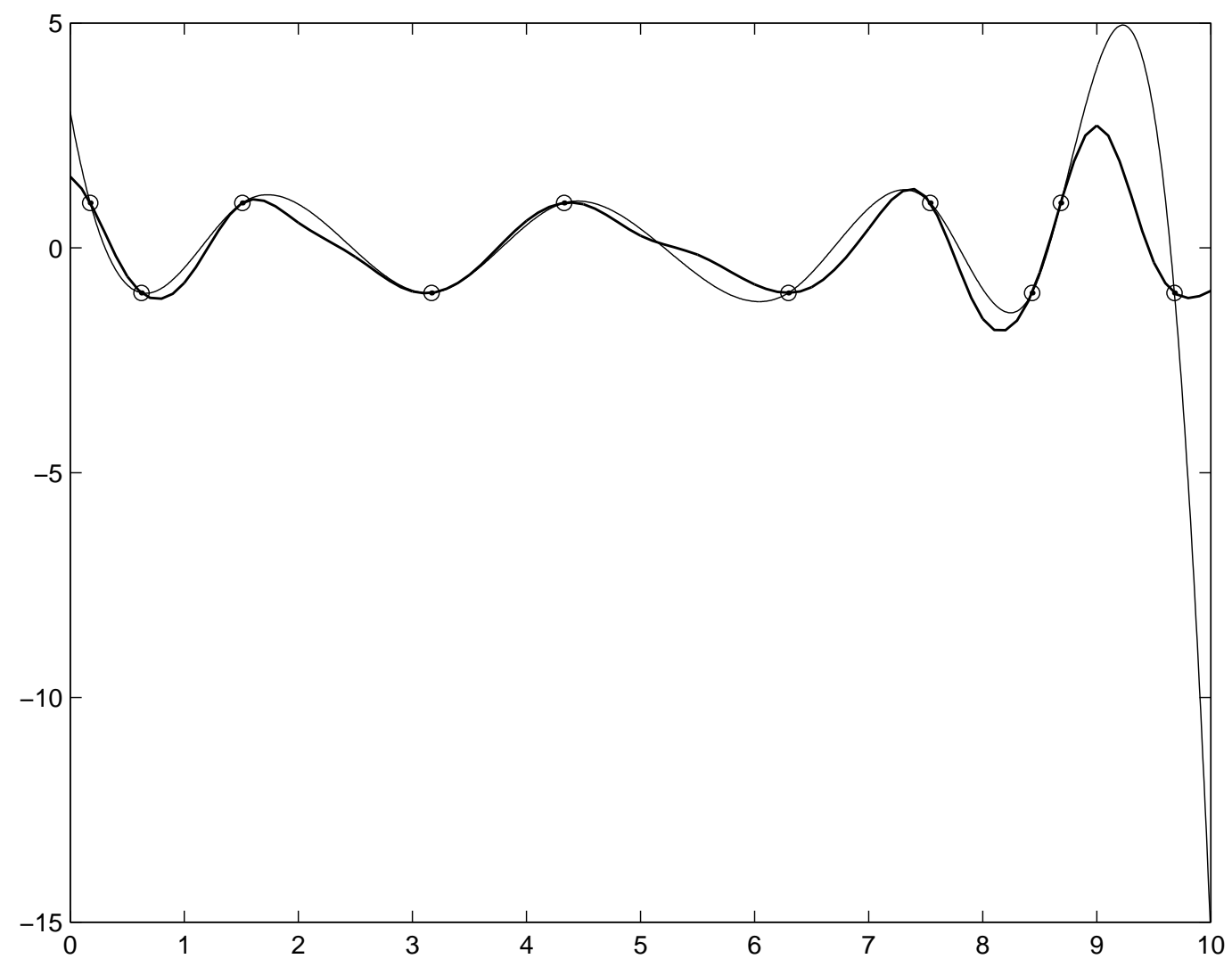

Fig. 4.3. The multilevel cubic spline interpolant of Fig. 4.1. for ground level $S_{M}(2 \cdot)$, together with the cubic spline interpolant to the same (rapidly varying) data with the not-a-knot end condition (thin line). Notice the differing behaviour at the endpoints. 
Example (Cubic spline end conditions). Consider interpolation to

$$
\Theta:=\{1,2, \ldots, n\}
$$

from the space $S_{M}$ generated by the cubic spline $M$. Let $M_{j}$ denote the cubic B-spline centred at $j$. Then the representor of point evaluation at $j$ can be written as

$$
R_{j}=\frac{1}{6} M_{j-1}+\frac{2}{3} M_{j}+\frac{1}{6} M_{j+1}
$$

Here

$$
\operatorname{supp} M_{j}=[j-2, j+2], \quad \operatorname{supp} R_{j}=[j-3, j+3] .
$$

As remarked at the end of Section 3, interpolation from $V:=\operatorname{span}\left\{R_{1}, \ldots, R_{n}\right\}$ to $\Theta$ is correct. For $n \geq 4$ this spline space has the following basis

$\frac{4}{19} M_{0}+\frac{15}{19} M_{1}, \frac{1}{5} M_{1}+\frac{4}{5} M_{2}, M_{3}, M_{4}, \ldots M_{n-3}, M_{n-2}, \frac{4}{5} M_{n-1}+\frac{1}{5} M_{n}, \frac{15}{19} M_{n}+\frac{4}{19} M_{n+1}$.

The Gramian matrix for the basis $R_{1}, \ldots, R_{n}$ is the diagonally dominant bidiagonal matrix

$$
\left[R_{j}(i)\right]_{i, j=1}^{n}=\frac{1}{36}\left[\begin{array}{ccccccc}
18 & 8 & 1 & & & & \\
8 & 18 & 8 & 1 & & & \\
1 & 8 & 18 & 8 & 1 & & \\
& \ddots & \ddots & \ddots & \ddots & \ddots & \\
& & 1 & 8 & 18 & 8 & 1 \\
& & & 1 & 8 & 18 & 8 \\
& & & & 1 & 8 & 18
\end{array}\right] .
$$

If the interpolant is written as $s=\sum_{j=0}^{n+1} a_{j} M_{j}$, then the coefficients can be found by solving the diagonally dominant tridiagonal system

$$
\begin{aligned}
15 a_{0}-4 a_{1}+a_{2} & =0, \\
\frac{1}{6} a_{j-1}+\frac{2}{3} a_{j}+\frac{1}{6} a_{j+1} & =f(j), \quad j=1, \ldots, n, \\
a_{n-1}-4 a_{n}+15 a_{n+1} & =0 .
\end{aligned}
$$

The cubic spline end condition implied by (4.1) is not any of those given in [B78:IV]. By way of comparison, the "natural" and not-a-knot end conditions for the left end point are

$$
s^{\prime \prime}(1)=a_{0}-2 a_{1}+a_{2}=0, \quad s^{\prime \prime \prime}(2+)-s^{\prime \prime \prime}(2-)=a_{0}-4 a_{1}+6 a_{2}-4 a_{3}+a_{4}=0,
$$

respectively. 


\subsection{Box spline example}

We now give examples our scheme for the $M_{222}$ box spline. For the interpolation points $\Theta$ we take $[0,4]^{2} \cap \mathbb{Z}^{2}$ with the point $(1,1)$ replaced by $(1 / 2,1 / 2)$.
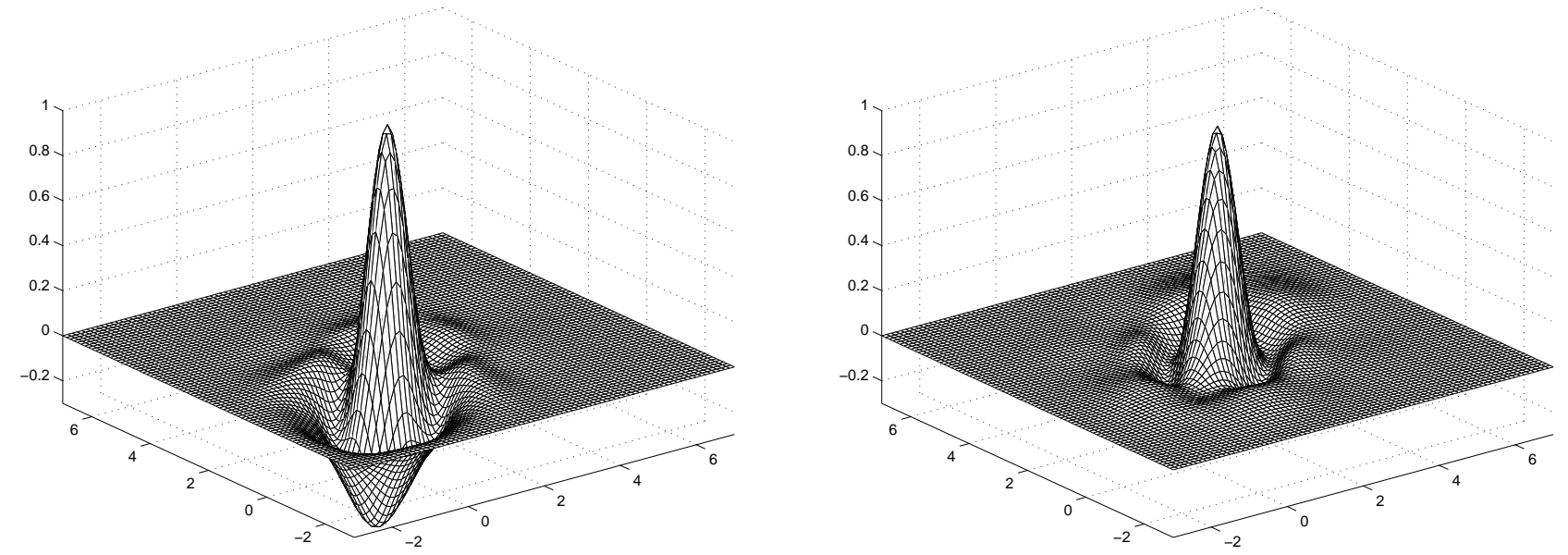

Fig. 4.4. Lagrange functions for interpolation on the grid $\Theta$. The first is for the point $(1 / 2,1 / 2)$, and the second for the point $(2,2)$.

\section{References}

[B78] C. de Boor, "A Practical Guide to Splines", Springer Verlag, New York, 1978.

[B94] C. de Boor, Gauss elimination by segments and multivariate polynomial interpolation, in "Approximation and Computation: A Festschrift in Honor of Walter Gautschi" (R. V. M. Zahar Ed.), pp. 1-22, ISNM 119, Birkhäuser Verlag, Basel-Boston-Berlin, 1994.

[BHR93] C. de Boor, K. Höllig, and S. D. Riemenschneider, "Box Splines", Springer-Verlag, New York, 1993.

[BR92] C. de Boor and A. Ron, The least solution for the polynomial interpolation problem, Math. Z. 210 (1992), 347-378.

[CW92] C. K. Chui and Jianzhong Wang, On compactly supported spline wavelets and a duality principle, Trans. Amer. Math. Soc. 330 (1992), 903-915.

[J99] M. J. Johnson, Scattered data interpolation from principal shift-invariant spaces, Preprint, 1999.

[LLS97] W. Lawton, S. L. Lee, and Zuowei Shen, Stability and orthonormality of multivariate refinable functions, SIAM J. Math. Anal. 28(4) (1997), 999-1014.

[RS92] S.D. Riemenschneider and Zuowei Shen, Wavelets and prewavelets in low dimensions, J. Approx. Theory $\mathbf{7 1}$ (1992), 18-38.

[RS95] S. D. Riemenschneider and Zuowei Shen, General interpolation on the lattice $h \mathbb{Z}^{s}$ : compactly supported fundamental solutions, Numer. Math. 70 (1995), 18-38.

[W99] S. Waldron, Degree reducing interpolation in inner product spaces, preprint, 1999. 